American Journal of Applied Sciences 8 (4): 374-381, 2011

ISSN 1546-9239

(C) 2010 Science Publications

\title{
Particle Swarm Optimization Approach for Optimal Design of Switched Reluctance Machine
}

\author{
Mahadevan Balaji and Vijayarajan Kamaraj \\ Department of Electrical and Electronics Engineering, \\ Sri Sivasubramaniya Nadar College of Engineering, Chennai, India
}

\begin{abstract}
Problem statement: Switched Reluctance Motors (SRMs) are widely used in various applications due to their inherent simplicity and rugged construction In SRM, torque output and torque ripple are sensitive to stator and rotor pole arcs and their selection is a vital part of SRM design process. In this study Particle Swarm Optimization technique is proposed for determining optimum pole arc of SRM. Approach: The problem of determining optimum pole arc is formulated as a multiobjective optimization problem with the objective of maximizing average torque and minimizing torque ripple. A comprehensive program based on analytical model is developed in Matlab to compute the value of inductance and average torque. Results: The optimization procedure is tested on 8/6, four-phase, $5 \mathrm{HP}, 1500 \mathrm{rpm}$ SRM. The results are compared and investigated with those obtained from Genetic Algorithm (GA) technique and Finite Element Analysis(FEA) simulation. Conclusion: The results demonstrate that the proposed method is effective and outperforms GA in terms of solution quality, accuracy, constraint handling.
\end{abstract}

Key words: Average torque, genetic algorithm, particle swarm optimization, switched reluctance machine, torque ripple, finite element analysis

\section{INTRODUCTION}

Simple and robust structure, high efficiency and fault tolerability of Switched Reluctance Machine (SRM) are good reasons for its selection in variable speed applications (Lawrenson et al., 1980). The output power of an SRM is higher than that of a comparable induction motor and the torque-inertia ratio is also higher due to the absence of rotor windings (Husain and Ehsani, 1994). Each stator pole has a simple winding, which is usually concentric. Suitable windings are connected together to form the motor phases. This simplicity gives the SRM the possibility of operating at very high speeds with a high output and a better mechanical acceleration (Miller, 1989). The main disadvantage of SRM is higher torque ripple which contributes to acoustic noise and vibration. The torque pulsation in SRM is due to highly non-linear and discrete nature of torque production mechanism (Husain and Ehsani, 1994).In recent years to achieve efficient design of electrical machines, researchers have focused on computer-aided electromagnetic design approach and evolutionary programming approach. Sahin et al., 2000, has discussed an approach to determine optimum geometry of SRM with minimum torque ripple. Generalized regression neural network based optimization of SRM with the objective of maximizing average torque and minimizing torque ripple is discussed in (Sahraoui, et al., 2007).Optimization techniques like Genetic Algorithm and Taguchi algorithm have been applied for switched reluctance machine design(Kano et al., 2010; Mirzaeian et al., 2002; Nabeta et al., 2008). From the literature it is evident that computational intelligence techniques like genetic algorithm and artificial neural network have been successfully applied for design optimization of SRM. In recent years, several heuristic optimization techniques such as ant Colony Algorithm (ACO), PSO (Roomi et al., 2010, Xiang et al., 2009)are applied to solve a variety of complex engineering problems. In this work an attempt has been made to apply Particle Swarm Optimization (PSO), for design optimization of SRM. The focus of this work is pole arc optimization of SRM with the objective of maximizing average torque and minimizing torque ripple using PSO approach. The PSO (Kennedy and Eberhart, 1995; Wang et al., 2008; Eberhart and Kennedy, 1995; Eberhart and Shi, 2000; Chaturvedi et al., 2009; Clerc and Kennedy, 2002) algorithm is one of the modern evolutionary algorithms. This algorithm was first proposed by Kennedy and Eberhart (1995). PSO is a population-based search algorithm characterized as conceptually simple, easy to

Corresponding Author: Mahadevan Balaji, Department of Electrical and Electronics Engineering, Sri Sivasubramaniya Nadar College of Engineering, Chennai, India 
implement and computationally efficient. Similar to the other population-based evolutionary algorithms PSO is initialized with a population of random solutions (Coelho et al., 2009). Unlike the most of the evolutionary algorithms, each potential solution in PSO is also associated with a randomized velocity and the potential solutions called particles, are then "flown", through the problem space.The performance of PSO algorithm is compared with GA based optimization. The results show that PSO based approach performs better in terms of solution quality, accuracy and convergence time.

Design optimization of SRM- Problem Formulation: The optimal design problem can be formulated as the following multiobjective nonlinear optimization problem:

$\min _{x \in F} f_{1}(x), f_{2}(x), \ldots, f_{k}(x)$

where, $x \in R^{n} f_{i}: R^{n} \mapsto R$ and $F$ is the feasible set of problem (1) which is described by the inequalities as follows:

$$
F=\left\{x \in R^{n}: g_{i}(x) \leq 0, i=1,2, \ldots, p\right\}
$$

where, $\mathrm{g}_{\mathrm{i}}(\mathrm{x})$ is called the constraint function .We denote $f(x) \in R^{k}$ the vector made up of all objective functions, that is:

$$
f(x)=\left(f_{1}(x), f_{2}(x), \ldots . . f_{k}(x)\right)
$$

Pareto optimal solution: An ideal solution of (1) would be a point $\mathrm{x}^{*} \in \mathrm{F}$ such that:

$$
\mathrm{f}_{\mathrm{i}}\left(\mathrm{x}^{*}\right) \leq \mathrm{f}_{\mathrm{i}}(\mathrm{x}), \forall \mathrm{x} \in \mathrm{F}, \forall \mathrm{i} \in\{1,2, \ldots . . \mathrm{k}\}
$$

The point $\mathrm{x}^{*}$ seldom exists, therefore(1) turns into finding some or all the pareto optimal solutions. A point $x^{*} \in \mathrm{F}$ is a pareto optimal solution of (1) if there does not exist any feasible point $x \in F$ such that:

$$
\mathrm{f}_{\mathrm{i}}(\mathrm{x}) \leq \mathrm{f}_{\mathrm{i}}\left(\mathrm{x}^{*}\right), \forall \mathrm{i} \in\{1,2, \ldots . . \mathrm{k}\}
$$

And:

$$
f_{j}(x) \leq f_{j}\left(x^{*}\right)
$$

for at least one index $\mathrm{j} \in\{1,2, ., \mathrm{k}\}$

There exists a wide variety of methods that can be used to compute Pareto optimal solutions. A widely used technique consists of reducing the multiobjective problem given by Eq. 1 to a single objective one by means of "scalarization" procedure. The "scalarization" procedure in this study consists of assigning each objective function a cost coefficient and then minimizing the function obtained by summing up all the objective functions scaled by their cost coefficients (Liuzzi et al., 2003), that is:

$$
\min _{x \in F} \sum_{i=1}^{k} c^{i} f_{i}(x)
$$

The global solution of the problem is affected by the coefficient $c^{i}$. The cost coefficient is determined by the following equation:

$$
\begin{aligned}
& z_{i}^{*}=\min _{x \in F} f_{i}(x) \\
& c^{i}=\frac{1}{z_{i}^{*}}
\end{aligned}
$$

Design variables: The structure of $8 / 6 \mathrm{SRM}$ is presented in Fig. 1. The torque characteristics of SRM depend on number of poles, number of phases, statorrotor pole overlap angle and pole geometry (Husain, 2002). From the literature (Arumugam et al., 1988; Koibucuchi et al., 1997) it is evident that torque output and torque ripple are sensitive to stator and rotor pole arcs and their choice is a critical part of SRM design process. The choice of pole arcs depends on the application and there is no distinct value that is suitable for all applications. Further optimum pole arcs are a compromise between various conflicting requirements (Miller, 1989). Hence in this study the two most significant parameters on average torque and torque ripple are taken as design variables:

$\mathrm{X}_{1}$ Rotor Pole arc $\left(\beta_{\mathrm{r}}\right)$

$\mathrm{X}_{1}$ Stator pole arc. $\left(\beta_{\mathrm{r}}\right)$

The remaining design parameters are treated as fixed for the optimization process.

$F(x)=-\left(A * f_{1}(x)+B * f_{2}(x)\right)$

Where:

$\mathrm{f}_{1}(\mathrm{x})=$ Maximization of average torque.

$\mathrm{f}_{2}(\mathrm{x}=$ Maximization of inductance ratio 


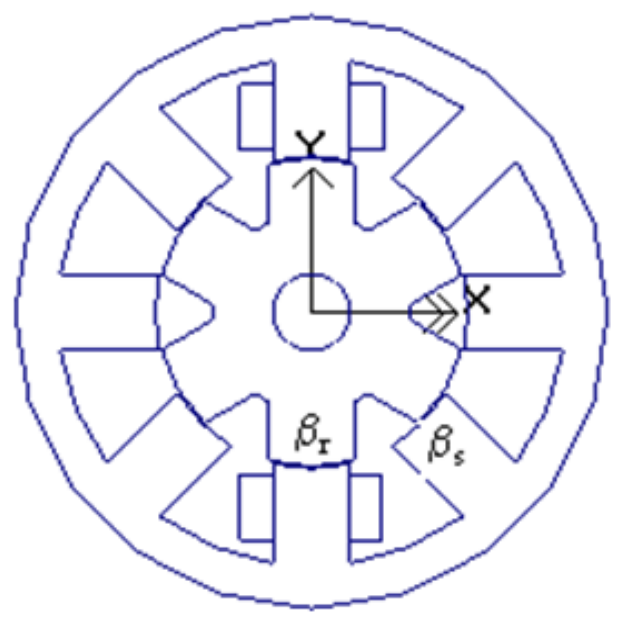

Fig. 1: Schematic diagram of $8 / 6$ SRM

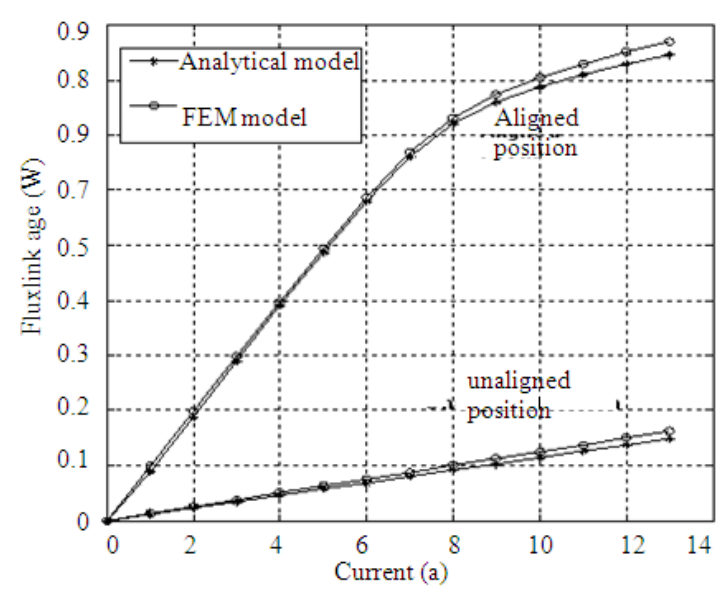

Fig. 2: Plot of Flux Linkage Vs Current

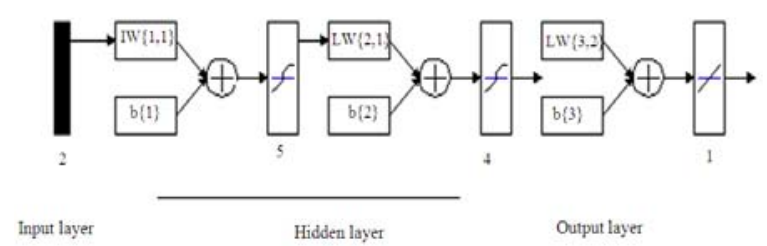

Fig. 3: Neural Network Structure

Objective function: The multiobjective problem formulation is given by:

The constants $\mathrm{A}$ and $\mathrm{B}$ are determined using the "scalarization" procedure. In view of the fact that the average torque and inductance ratio of the motor is to be maximized, minus sign is introduced in the fitness function.
Calculation of average torque: Finite-Element Method (FEM) (Arkadan and Kielagas, 1994), Magnetic Equivalent Circuit (MEC) method (Moallem and Dawson, 1998) and piecewise linear model (Miller and Mcglip, 1990) are reported for the analysis of SRM. In this study analytical method described is used to determine the inductance and average torque of the machine.

The average torque is given by:

$\mathrm{Tav}=\frac{\delta \mathrm{WmN}_{\mathrm{s}} \mathrm{N}_{\mathrm{r}}}{4 \pi}$

$\delta \mathrm{Wm}=\mathrm{Wm}$ 'aligned $-\mathrm{Wm}$ 'unaligned

$=\Delta \mathrm{i}\left(\lambda_{1}+\lambda_{2}+\ldots \ldots \frac{1}{2} \lambda_{\mathrm{n}}\right)-\frac{1}{2} \lambda_{\mathrm{u}} \mathrm{I}_{\mathrm{p}}$

where, $\Delta i=\frac{I_{p}}{n}$.

A comprehensive program is written in MATLAB to compute the difference of co energies at aligned and unaligned position. The aligned coenergy is calculated with trapezoidal integration algorithm. Once $\delta w m$ is determined, the average torque is calculated using Eq. 10. The results obtained are compared and validated with FEA model. The design data for validating the analytical model is given in appendix1. The flux linkage-current characteristics obtained by analytical model and FEM model are presented in Fig. 2. Table 1 presents the torque value computed by two methods. The closeness of the results have confirmed and validated the analytical model.

For optimal design of the SR motor by evolutionary algorithms such as PSO, a large number of performance evaluations are required and the computational time by analytical method would be very large. Therefore, in this study, an Artificial Neural Network (ANN) based expert system for performance prediction of the SR motor has been developed. The ANN expert system can be used as a very fast performance prediction tool in design optimizing programs. A feed forward neural network as shown in Fig. 3 is trained with discrete points in the design domain considering the rules of feasible triangle (Lawrenson et al., 1980).

The stator and rotor pole arc form the input to the network and the torque obtained by analytical computation form the output. Backpropoagation algorithm is used to train the neural network. The results of the network are satisfactory when tested with 
Am. J. Applied Sci., 8 (4): 374-381, 2011

Table 1: Computed average torque by analytical and FEM method

\begin{tabular}{lll}
\hline & Analytical value & FEM calculation \\
\hline Average Torque & $23.14 \mathrm{Nm}$ & $23.61 \mathrm{Nm}$ \\
\hline
\end{tabular}

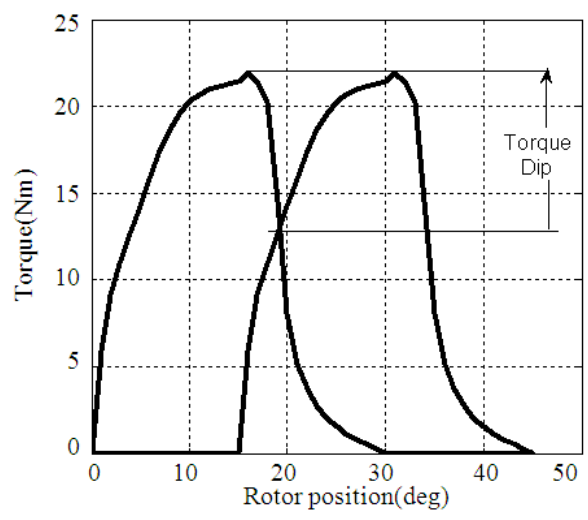

Fig. 4: Torque vs rotor position characteristics

data which were not subjected to training. This network is incorporated in the optimization routine to compute the value of average torque.

Evaluation of torque ripple: The majority of torque ripple occurs in the phase overlap region, where the torque producing responsibility is commutated from one phase to another. The extent of design dependent overlap sets the limit on the electronic controller on effective torque sharing between two adjacent phases to minimize torque ripple. Torque ripple expected from $\mathrm{SRM}$ is evaluated from the torque dips in $\mathrm{T}-\mathrm{i}-\theta$ characteristics. Torque dip is the difference between the peak torque of a phase and the torque at an angle where two overlapping phases produce equal torque at equal levels of current. This is due to the insufficiency of the incoming phase in supplying the required torque in those rotor positions (Husain, 2002). Figure 4 shows the torque dip present in the initial design. The effect of pole arc variation on mean torque and torque dip can be evaluated from inductance overlap ratio $\mathrm{K}_{\mathrm{L}}$ given by Eq. 18. Inductance overlap ratio gives a direct measure of torque overlap of adjacent phases:

$$
\mathrm{K}_{\mathrm{L}}=1-\frac{\varepsilon}{\min \left(\beta_{\mathrm{s}}, \beta_{\mathrm{r}}\right)}
$$

From Eq. 18 it is evident that by widening the stator and rotor poles, torque overlap can be increased. The higher the $\mathrm{K}_{\mathrm{L}}$, the lower will be the torque dip and the higher will be the mean torque as well. Since the inductance ratio has to be maximized the fitness function is taken as minus of $\mathrm{K}_{\mathrm{L}}$.

Design constraints: The following are the constraints are imposed on the design optimization problem according to the rules of feasible triangle (Lawrenson et al., 1980).

$$
\begin{aligned}
& \mathrm{x}_{1} \geq \mathrm{x}_{2} \\
& \frac{2 \pi}{\mathrm{Nr}}-\mathrm{x}_{1} \succ \mathrm{x}_{2} \\
& \mathrm{x}_{2} \succ \varepsilon
\end{aligned}
$$

To have a practically feasible and acceptable final design the following performance constraints are imposed:

- Average torque should be greater than $21 \mathrm{~N}-\mathrm{m}$

- Clearance space between the tips of windings should be greater than $5 \mathrm{~mm}$

The constraints are taken into account by penalizing the fitness proportionally to the constraint violations.

Overview of PSO: PSO originally developed by Kennedy and Eberhart (1995) is a population based swarm algorithm. Swarm intelligence is an emergent research area with populational and evolutionary characteristics similar to those of genetic algorithms. In PSO, a number of particles form a "swarm" that evolve or fly throughout the feasible hyperspace to search for fruitful regions in which optimal solution may exist. Each particle has two vectors associated with it, the position $\left(\mathrm{X}_{\mathrm{i}}\right)$ and velocity $\left(\mathrm{V}_{\mathrm{i}}\right)$ vectors. In $\mathrm{N}$ dimensional search space, $\mathrm{X}_{\mathrm{i}}=\left[\mathrm{x}_{\mathrm{i} 1}, \mathrm{x}_{\mathrm{i} 2}, \ldots, \mathrm{x}_{\mathrm{iN}}\right]$ and $\mathrm{V}_{\mathrm{i}}$ $=\left[\mathrm{v}_{\mathrm{i} 1}, \mathrm{v}_{\mathrm{i} 2}, \ldots, \mathrm{v}_{\mathrm{iN}}\right]$ are the two vectors associated with each particle i. During their search, members of the swarm interact with each others in a certain way to optimize their search experience. There are different variants of particle swarm paradigms but the most commonly used one is the gbest model where the whole population is considered as a single neighborhood throughout the flying experience (Chaturvedi et al., 2009; Clerc and Kennedy, 2002). In each iteration, particle with the best solution shares its position coordinates (gbest) information with the rest of the swarm. Each particle updates its coordinates based on its own best search experience (pbest) and gbest according to the following equations: 
Am. J. Applied Sci., 8 (4): 374-381, 2011

$$
\begin{aligned}
& \mathrm{v}_{\mathrm{i}}^{\mathrm{k}+1}=\mathrm{wv}_{\mathrm{i}}^{\mathrm{k}}+\mathrm{c}_{1} \operatorname{rand}_{1}\left(\text { pbest }_{\mathrm{i}}^{\mathrm{k}}-\mathrm{x}_{\mathrm{i}}^{\mathrm{k}}\right) \\
& +\mathrm{c}_{2} \operatorname{rand}_{2}\left(\text { gbest }_{\mathrm{i}}^{\mathrm{k}}-\mathrm{x}_{\mathrm{i}}^{\mathrm{k}}\right) \\
& \mathrm{x}_{\mathrm{i}}^{\mathrm{k}+1}=\mathrm{x}_{\mathrm{i}}^{\mathrm{k}}+\mathrm{v}_{\mathrm{i}}^{\mathrm{k}+1}
\end{aligned}
$$

where, $\mathrm{c}_{1}$ and $\mathrm{c}_{2}$ are two positive acceleration constants, they keep balance between the particle's individual and social behavior when they are set equal; $\operatorname{rand}_{1}$ and $\operatorname{rand}_{2}$ are two randomly generated numbers with a range of $[0$, 1] added in the model to introduce stochastic nature in particle's movement; and $\mathrm{w}$ is the inertia weight and it keeps a balance between exploration and exploitation. In our case, it is a linearly decreasing function of the iteration index:

$\mathrm{w}(\mathrm{k})=\mathrm{w}_{\text {max }}-\left(\frac{\mathrm{w}_{\text {max }}-\mathrm{w}_{\text {min }}}{\text { iter }_{\text {max }}}\right) \times$ iter

Where:

$$
\begin{aligned}
& \text { iter }_{\max }=\text { The maximum number of iteration } \\
& \text { iter }=\text { The current iteration number } \\
& \mathrm{W}_{\max }=\text { The initial weight } \\
& \mathrm{w}_{\min }=\text { The final weight. }
\end{aligned}
$$

In conclusion, an initial value of $\mathrm{w}$ around 1 , with a gradual decline toward 0 isconsidered as a proper choice. The most important factor that governs the PSO performance in its search for optimal solution is to maintain a balance between exploration and exploitation. Exploration is the PSO ability to cover and explore different areas in the feasible search space while exploitation is the ability to concentrate only on promising areas in the search space and to enhance the quality of potential solution in the fruitful region. Exploration requires bigger step sizes at the beginning of the optimization process to determine the most promising areas then the step size is reduced to focus only on that area. This balanced is usually achieved through proper tuning of PSO key parameters. Recently, PSO developments and applications have been widely explored in engineering and science mainly due to its distinct favorable characteristics (Chaturvedi et al., 2009). Just like in the case of other evolutionary algorithms, PSO has many key features that attracted many researchers to employ it in different applications in which conventional optimization algorithms might fail such as:

- It only requires a fitness function to measure the "quality" of a solution instead of complex mathematical operations like gradient, Hessian, or matrix inversion. This reduces the computational complexity and relieves some of the restrictions that are usually imposed on the objective function like differentiability, continuity, or convexity
- It is less sensitive to a good initial solution since it is a population based method

- It can be easily incorporated with other optimization tools to form hybrid ones

- It has the ability to escape local minima since it follows probabilistic transition rules

More interesting PSO advantages can be emphasized when compared to other members of evolutionary algorithms like:

- It can be easily programmed and modified with basic mathematical and logic operations. It is inexpensive in terms of computation time and memory

- It requires less parameter tuning.

- It works with direct real valued numbers that eliminates the need to do binary conversion of classical canonical genetic algorithm

Implementation of PSO for Optimal Design of SRM: The algorithm for PSO based SRM design optimization can be summarized as follows:

Step1: Initialize PSO parameters such as $\mathrm{w}_{\max }, \mathrm{w}_{\min }$, $\mathrm{c}_{1}, \mathrm{c}_{2}$ and Iter $_{\text {max }}$

Step2: Generate initial population of $\mathrm{N}$ particles with random positions and velocities.

Step3: Evaluate the fitness of each particle using Eq. 9

Step4: Update personal best: Compare the fitness value of each particle with its pbests. If the current value is better than pbest, then set pbest value to the current value.

Step5: Update global best: Compare the fitness value of each particle with gbest. If the current value is better than gbest, set gbest to the current particle's value.

Step6: Update weight: Calculate weight $\mathrm{W}^{\mathrm{k}+1}$ using Eq. 18

Step7: Update velocities: Calculate velocities $\mathrm{V}^{\mathrm{k}+1}$ using Eq. 16

Step8: Update positions: Calculate positions $\mathrm{X}^{\mathrm{k}+1}$ using Eq. 17

Step9: Return to step (4) until the current iteration reaches the maximum iteration number

Step10: Output the optimal design variables.

\section{RESULTS}

The proposed PSO technique for multi-objective design optimization of SRM is tested on a 5HP motor. The specifications of the sample motors are given in Appendix 1.Acomparative study with GA was done to verify the Performance of the proposed algorithm. PSO and GA Parameters used for the simulation are 
Am. J. Applied Sci., 8 (4): 374-381, 2011

Table 2: Simulation parameters

\begin{tabular}{ll}
\hline GA & PSO \\
\hline Population size: 30 & Number of Particles: 30 \\
Generations: 50 & Iterations: 50 \\
Crossover rate: 0.8 & $\mathrm{C}_{1}=\mathrm{C}_{2}=2$ \\
Mutation rate: 0.1 & $\mathrm{~W}_{\max }=0.9$ \\
$\mathrm{~W}_{\min }=0.4$ & \\
\hline
\end{tabular}

summarized in Table 2. For the implementation of PSO, several parameters are required to be specified, such as acceleration factors ( $\mathrm{C} 1$ and $\mathrm{C} 2)$, Weighting factor $(\mathrm{W})$ and swarm size and termination criteria.

These parameters should be selected carefully for efficient performance of PSO. According to past experience the acceleration constants are set to be 2.0 Suitable choice of Weighting factor (W) provides a balance between the global and the local explorations, thus requiring less iteration on average to find as sufficiently optimal solution. In this study W decreases linearly from about $0.9-0.4$ as discussed in (Kennedy and Eberhart, 1995). The swarm size and maximum number of generations are fixed by trial and error method. The optimization procedure is terminated when 50 generations are reached.

\section{DISCUSSION}

\section{Comparative studies:}

Solution quality: The results obtained from PSO and GA based design methods are given in Table 3. The convergence characteristics of GA and PSO methods for the sample motor are shown in Fig. 5. From the results it is seen that the PSO converges quickly and explore higher quality solution than the GA.

Robustness: To test the robustness of GA and PSO, 20 independent trials were carried out. The obtained results by each method are given in Fig. 6. From the Figure, it is clear that the minimum, average and standard deviations obtained by PSO are better than GA.

Characterization using FEA: The optimized geometry was exposed to finite-element calculation. The flux lines at aligned position is shown in Fig. 7. The static torque characteristics of the optimal machine at rated current of $13 \mathrm{~A}$ is shown in Fig. 8. The optimal machine produced an average torque of $29.19 \mathrm{Nm}$ with a torque dip of $5.7 \mathrm{Nm}$. The results of finite-element calculation confirm the application of optimization procedure for SRM design.
Table 3: Results of optimal design

\begin{tabular}{lcll}
\hline & & \multicolumn{3}{l}{ Optimal design } & \\
& Initial design & PSO & GA \\
\hline Stator Pole arc & $18.0000 \mathrm{deg}$ & $21.910 \mathrm{deg}$ & $21.740 \mathrm{deg}$ \\
Rotor Pole arc & $22.0000 \mathrm{deg}$ & $22.910 \mathrm{deg}$ & $22.770 \mathrm{deg}$ \\
Average Torque & $22.9800 \mathrm{Nm}$ & $29.670 \mathrm{Nm}$ & $29.400 \mathrm{Nm}$ \\
Inductance ratio & 0.1667 & 0.315 & 0.310 \\
Torque dip & $8.8400 \mathrm{Nm}$ & $5.770 \mathrm{Nm}$ & $5.800 \mathrm{Nm}$ \\
\hline
\end{tabular}

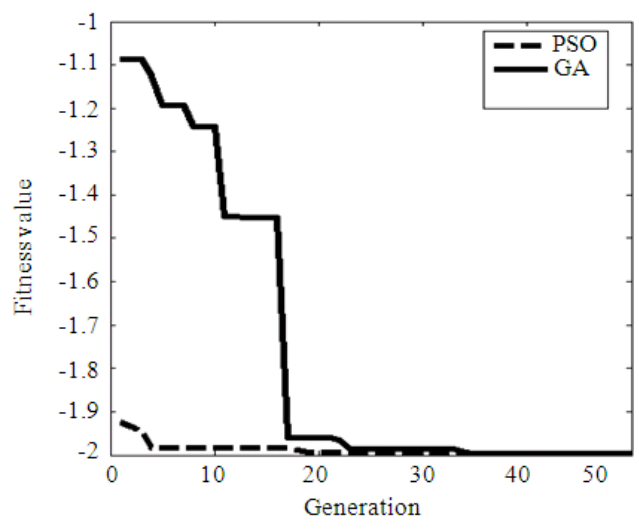

Fig. 5: Comparison of convergence characteristics of GA and PSO based methods

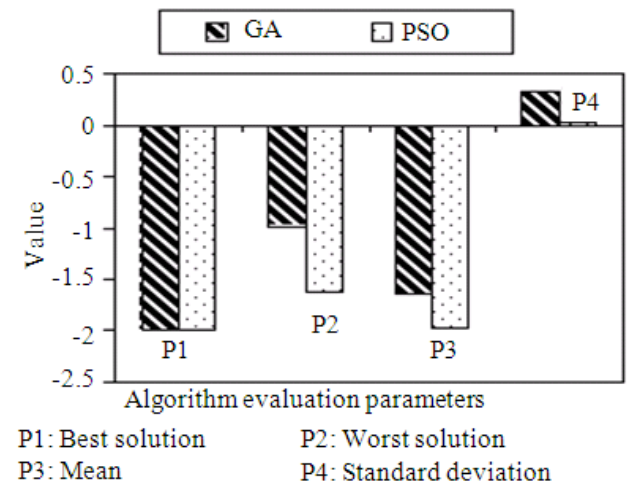

Fig.6: Comparison of PSO and GA methods

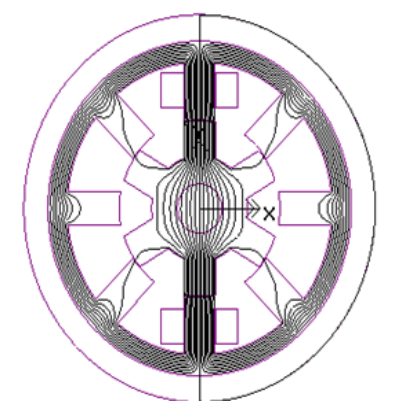

Fig. 7: Flux Lines at aligned position 


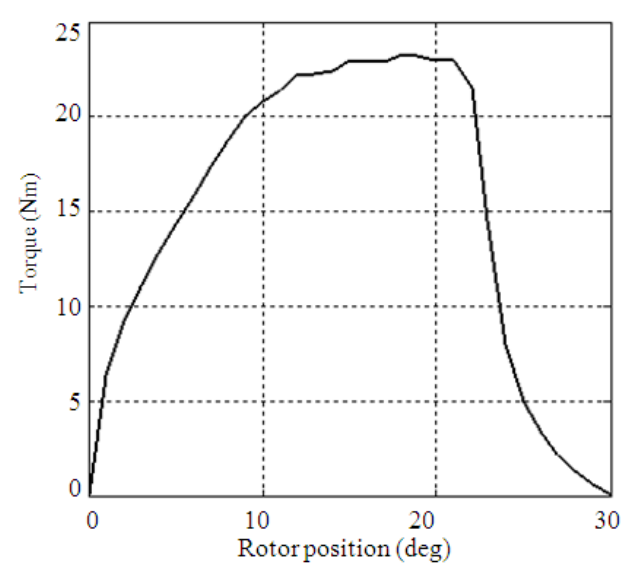

Fig. 8: Static torque characteristics

\section{CONCLUSION}

This study describes the design optimization procedure of SRM using PSO with the objective of maximizing average torque and minimizing torque ripple. ANN based expert system is developed for faster performance prediction during the optimization process. The results obtained by this approach show improvement in average torque and reduction in torque dip. The optimized geometry was exposed to finiteelement calculation using MagNet software. The results of finite-element calculation validate the application of PSO based optimization procedure for SRM design.. The PSO based method is a promising tool for solving design optimization problems of Switched Reluctance Motor.

\section{REFERENCES}

Arkadan, A.A. and B.W. Kielagas, 1994. Switched Reluctance motor drive systems dynamic performance prediction and experimental verification. IEEE Trans. Energy Conversion, 9: 36-44. DOI: $10.1109 / 60.282474$

Arumugam, R., J.F. Lindsay and R. Krishnan, 1988. Sensitivity of Pole arc/pole pitch ratio on switched reluctance motor performance. Proceedings of the IEEE Industry Applications Society Annual Meeting, Oct. 2-7, IEEE Xplore, Pittsburgh, PA., pp: 50-54. DOI: 10.1109/IAS.1988.25041

Chaturvedi, K.T., M. Pandit and L. Srivastava, 2009. Particle swarm optimization with time varying acceleration coefficients for non-convex economic power dispatch. Int. J. Elec. Power Energy Syst., 31: 249-257. DOI: 10.1016/j.ijepes.2009.01.010
Clerc, M. and J. Kennedy, 2002. The particle swarm explosion, stability, and convergence in a multidimensional complex space: IEEE Trans. Evolutionary Comput., 6: 58-73. DOI: 10.1109/4235.985692

Eberhart, R. and J. Kennedy, 1995. A new optimizer using particle swarm theory. Proceedings of the Sixth International Symposium on Micro Machine and Human Science, Oct. 4-6, IEEE Xplore, Japan, pp: 39-43. DOI: 10.1109/MHS.1995.494215

Eberhart, R.C. and Y. Shi, 2000. Comparing inertia weights and constriction factors in particle swarm optimization. Proceedings of the IEEE International Congress on Evolutionary Computation, San Diego, July 16-19, IEEE Xplore, USA., pp: 84-88. DOI: 10.1109/CEC.2000.870279

Husain, I. and M. Ehsani, 1994. Torque ripple minimization in switched reluctance motor drives by PWM current control. IEEE Trans. Power Elect., 11: 83-88. DOI: 10.1109/63.484420

Husain, I., 2002. Minimization of Torque Ripple in SRM drives: IEEE Trans. Ind. Elect., 49: 28-39. DOI: $10.1109 / 41.982245$

Kano, Y., T. Kosaka and N. Matsui, 2010. Optimum design approach for a two-phase switched reluctance compressor drive. IEEE Trans. Ind. Appl., $\quad 46:$ 955-964. 10.1109/TIA.2010.2045212

Kennedy, J. and R. Eberhart, 1995. Particle swarm optimization. Proceedings of IEEE International Conference on Neural Networks, Nov. 27-1 Dec., IEEE Xplore, Australia, pp: 1942-1948. DOI: 10.1109/ICNN.1995.488968

Koibucuchi, K., T. Tohno and K. Sawa, 1997. A basic study for optimal design of switched reluctance motor by finite element method. IEEE Trans. Magnetics, 33: 2077-2080. DOI: 10.1109/20.582726

Lawrenson, P.J., J.M. Stephenson, N.N. Fulton, P.T. Blenkinsop and J. Corda, 1980. Variable-speed switched reluctance motors. Proc. Elec. Power Appl, 127: 253-265. DOI: 10.1049/ip-b:19800034

Liuzzi, G., S. Lucidi, F. Parasiliti and M. Villanier, 2003. Multiobjective optimization techniques for the design of induction motors. IEEE Trans. Magnetics, 39: 1261-1264. DOI: 10.1109/TMAG.2003.810193

Miller, T.J.E., 1989. Brushless Permanent-Magnet and Reluctance Motor Drives. 1st Edn., Clarendon Press,UK., ISBN-10: 0198593694, pp: 207.

Miller,T.J.E. and M. Mcglip,1990. Nonlinear theory of the Switched reluctance motor for rapid computeraided design. Inst. Elec. Eng, Proc., B, 137: 337-347. 
Mirzaeian, B., M. Moallem, V. Tahani and C. Lucas, 2002. Multiobjective optimization method based on a genetic algorithm for switched reluctance motor design. IEEE Trans. Magnetics, 38: 15241527. DOI: $10.1109 / 20.999126$

Moallem, M. and G.E. Dawson, 1998. An improved magnetic equivalent circuit method for predicting the characteristics of highly saturated electromagnetic devices. IEEE Trans. Magnetics, 34: 3632-3635. DOI: 10.1109/20.717858

Nabeta, S.I., I.E. Chabu, L. Lebensztajn, D.A.P. Correa and W.M. daSilva et al., 2008. Mitigation of the torque ripple of a switched reluctance motor through a multiobjective optimization. IEEE Trans. Magnetics, 44: 1018-1021. DOI: 10.1109/TMAG.2007.915137

Roomi, S.M.M., P.L.M. Karuppi, P. Rajesh and B.G. Revathi , 2010. A particle swarm optimization based edge preserving impulse noise filter. J. Comput. Sci., 6: 1014-1020. DOI: 10.3844/jcssp.2010.1014.1020
Sahin, F., H.B. Erta and K. Leblebicioglu, 2000. Optimum geometry for torque ripple minimization of switched reluctance motors. IEEE Tran. Energy Conversion, 15: 30-39. DOI: 10.1109/60.849113

Sahraoui, H., H. Zeroug, , H.A. Toliyat, 2007. Switched reluctance motor design using neural-network method with static finite-element simulation. IEEE Trans. Magnetics, 43: 4089-4095. DOI: 10.1109/TMAG.2007.907990

Wang, Y.B., X. Peng, B.Z. Wei, 2008. A new particle swarm optimization based auto-tuning of PID controller. Proceedings of IEEE International Conference on Machine Learning and Cybernetics, July 12-15, IEEE Xplore, Kunming, pp: 18181823. DOI: $10.1109 /$ ICMLC.2008.4620701

Xiang, X., D. Zhang, J. Qin and Y. Fu, 2009. Ant colony with genetic algorithm based on planar graph for multiple sequence alignment. Inform. Technol. J., 9: 274-281. 